\title{
Improving the Tone Mapping Operators by Using a Redefined Version of the Luminance Channel
}

\author{
Nikola Banić and Sven Lončarić \\ Image Processing Group \\ Department of Electronic Systems and Information Processing \\ Faculty of Electrical Engineering and Computing \\ University of Zagreb, Croatia \\ $\{$ nikola.banic, sven.loncaric $\} @ f e r . h r$ \\ http://www.fer.unizg.hr/ipg/
}

\begin{abstract}
Tone mapping operators (TMOs) convert high dynamic range (HDR) images to low dynamic range (LDR) images and are important because of the limitations of many standard display devices. Even though the quality of the resulting LDR image mostly depends on TMO parameter values, in this paper it is shown that it can be further improved by using alternative definitions of the luminance channel, which TMOs process. A new model of the luminance channel calculation that increases the resulting LDR image quality is also proposed. The main advantage of the new model is that the TMOs that produce results of lower quality can be made to produce results of significantly higher quality.
\end{abstract}

Keywords: HDR, image quality, LDR, luminance, Minkowski norm, tone mapping.

\section{Introduction}

Recent advances in imaging technologies are responsible for greater availability of images with high dynamic range (HDR), i.e., with a high ratio between the largest and the smallest light intensity in the image [16]. As many standard display devices can manage only low dynamic range (LDR) images, using tone mapping operators (TMOs) to convert HDR to LDR images is required. TMOs mostly operate on the image luminance channel intensities. They can be divided into global TMOs that process each intensity in the same way and local TMOs that process the intensities depending on their surrounding area. TMOs based on histogram adjustment [10], sigmoidal contrast enhancement [2] photographic practice [17, luminance gradient field manipulation [5], image detail layer processing [4, imitation of human response to light [3] [15] [11] have been proposed. Local TMOs usually give visually more appealing results, while global TMOs are faster [8] 9. An example of tone mapping a HDR image is shown in Fig. 1

The optimal values of TMO parameters are often image-dependent and in most cases their determination requires human intervention. The Tone Mapped 


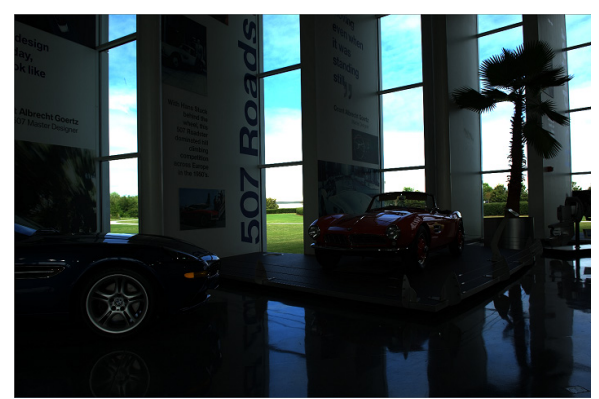

(a)

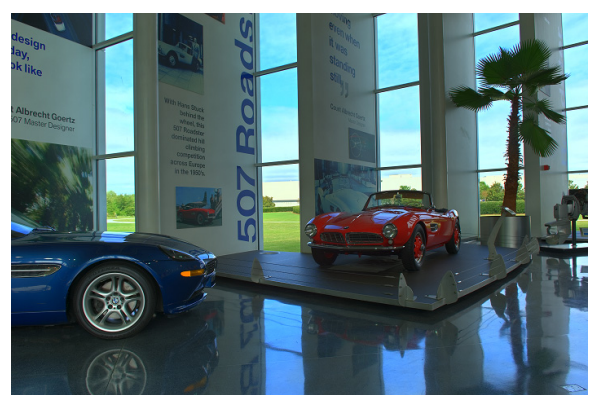

(b)

Fig. 1. Two examples of tone mapping of a HDR image: (a) linear mapping, (b) application of a TMO

image Quality Index (TMQI) was recently developed [18] in order to provide an objective measure of the quality of the tone mapped images and it was shown to be reasonably correlated with subjective evaluations of image quality. For the original HDR image and the tone mapped LDR image the TMQI is a number in interval $[0,1]$. It is based on the multiscale signal fidelity measure and the naturalness measure with a larger number corresponding to better quality. By using TMQI the subjective tests are avoided and it can be shown that together with TMO parameters the luminance channel definition can also increase the upper bound of the resulting image quality. The main contribution of this paper is the improvement of existing TMOs by using an alternative luminance channels.

The paper is structured as follows: In Section 2 it is shown that the definition of the luminance channel may affect the resulting image, in Section 3 a new model for the luminance channel is proposed, in Section 4 it is tested by means of measuring the TMQI, and the results are presented and discussed.

\section{The Luminance Channel}

TMOs usually operate in the luminance domain of the image. If the luminance value $L$ of the pixel $p=[R, G, B]^{T}$ becomes $L_{n e w}$ after TMO application, then in the resulting image the pixel $p$ is adjusted to become

$$
p_{n e w}=\frac{L_{n e w}}{L} p=\left[\frac{L_{n e w}}{L} R, \frac{L_{n e w}}{L} G, \frac{L_{n e w}}{L} B\right]^{T} .
$$

This shows that both the TMO and the calculation of $L$ have a role in creating the final result. The luminance channel TMO implementations often use [1] is the YUV colorspace Y channel also known as luma and defined as [7]

$$
L=0.299 R+0.587 G+0.114 B \text {. }
$$

Like the YUV colorspace, HSV, HSI, HSL, Lab, and other colorspaces also have their own definitions of luminance channels. For tone mapping any channel 


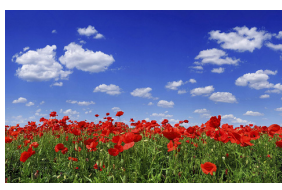

(a)

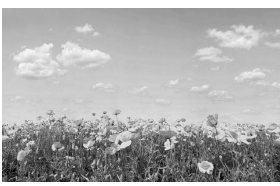

(e)

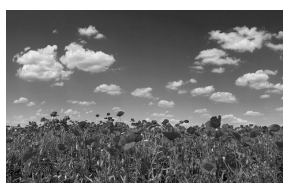

(b)

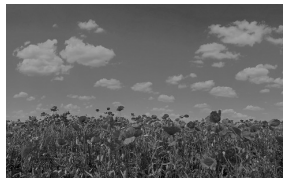

(f)

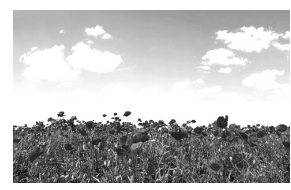

(c)

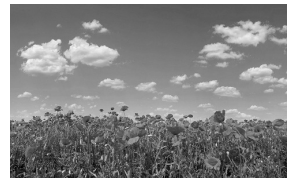

(g)

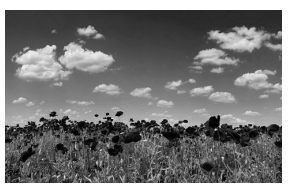

(d)

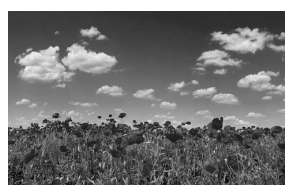

(h)

Fig. 2. Different channels for the same image that may assume the role of the luminance channel: (a) the original image, (b) Y of YUV, (c) first component of PCA, (d) G of RGB, (e) V of HSV, (f) I of HSI, (g) L of HSL, (h) L of Lab

may be used, even the one not defining luminance, e.g. the G of the RGB or the principal component of the PCA transform [13]. Fig. 22 shows different channels for the same image that may assume the role of the luminance channel. The obvious differences between channels may also lead to different result quality.

\section{A New Luminance Channel Definition}

All channels mentioned in the previous section are calculated in a fixed way. For all of them the value of $L$ for a given pixel depends only on its R, G, and B channels values, with exception of PCA, where all pixels are involved. All these channels are calculated in a fixed way and there are some calculation similarities like for the HSV's V and HSI's I channel:

$$
\begin{aligned}
& V=\max \{R, G, B\} . \\
& I=(R+G+B) / 3 .
\end{aligned}
$$

where the denominator can be ignored because in most cases for TMOs only the ratio between different intensities matters. Motivated by this similarity and in order to get a simply adjustable luminance channel, we propose a new definition:

$$
L=\left(R^{p}+G^{p}+B^{p}\right)^{\frac{1}{p}} .
$$

where $p$ is a parameter, which can be interpreted as the Minkowski norm. When $p \rightarrow \infty$, the Eq. 5 becomes the same as Eq. 3, and when $p=1$, then it effectively becomes the same as the HSI's I channel making the proposed definition a generalization of the two luminance channels. We note the extension of a TMO by the calculation of the Eq. [5] with an index $p$ so that $\mathrm{TMO}_{p}$ stands for TMO's application to the proposed channel with a specific value for parameter $p$. 
Table 1. Statistics of the best TMQIs for individual TMOs and luminance channels on 40 images (higher is better)

\begin{tabular}{|c|c|c||c|c||c|c|}
\hline TMO & \multicolumn{2}{|c||}{ Durand } & \multicolumn{2}{c||}{ Mantiuk } & \multicolumn{2}{c|}{ Drago } \\
\hline Luminance channel & mean & median & mean & median & mean & median \\
\hline Y of YUV (baseline) & 0.9124 & 0.9324 & 0.7286 & 0.7367 & 0.7521 & 0.7571 \\
\hline PCA & 0.8737 & 0.8885 & 0.7525 & 0.7622 & 0.7792 & 0.7758 \\
\hline G of RGB & 0.9043 & 0.9258 & 0.6875 & 0.7076 & 0.6832 & 0.7145 \\
\hline V of HSV & 0.9012 & 0.9169 & 0.7929 & 0.8097 & 0.8620 & 0.8630 \\
\hline I of HSI & 0.9124 & 0.9282 & 0.7541 & 0.7647 & 0.7798 & 0.7867 \\
\hline L of HSL & 0.9100 & 0.9256 & 0.7632 & 0.7726 & 0.7937 & 0.8080 \\
\hline L of Lab & 0.9110 & 0.9257 & 0.7220 & 0.7413 & 0.7446 & 0.7515 \\
\hline
\end{tabular}

\section{Experimental Results}

\subsection{Fixed Channels}

The impact of different channels in the role of the luminance channel on the resulting LDR image quality was tested by using the principal component of the PCA transform, the G channel of the RGB space, and the luminance channels of the YUV, HSV, HSI, HSL, and Lab color spaces. The Y channel of the YUV colorspace served as the baseline. The mentioned channels were processed by applying the OpenCV 3.0.0 C++ implementations [1] of Durand and Dorsey's local TMO [4, Mantiuk's gradient based TMO [11, and Drago's global TMO [3] representing different types of TMOs. From the HDR image set available at site of the NTUST Compute Graphics Group [14 20 images with the largest and 20 with the smallest dynamic range were selected for testing.

For each image and each luminance channel definition the best TMQI was chosen from TMQIs calculated for a large number of uniformly spread parameter value combinations. These combinations were designed to cover the majority of the TMO's practical parameter values. The large number of combinations is the reason why the test was performed for only 40 images. For Durand's operator the parameter values of the contrast and the two sigmas were from $\{0.5,1, \ldots, 25\}$ and $\{0.5,1, \ldots, 4\}$, respectively, and the values of Drago's operator bias parameter and Mantiuk's operator scale parameter from $\{0.01,0.02, \ldots, 1\}$. The results are summarized in Table 1 and they show a variation in the resulting LDR image quality depending on the used luminance channel definition.

The Jarque-Bera [6] test showed that the best TMQIs for a given TMO and luminance channel are not normally distributed, so instead of ANOVA [12], the Kruskal-Wallis (KW) non-parametric test [12] was used to test the significance of the mentioned differences. For Mantiuk's and Drago's TMO the $p$-value was below 0.01 proving that using different luminance channels has a $99 \%$ statistically significant impact on their best resulting image quality. Fig. 3 shows Drago's 


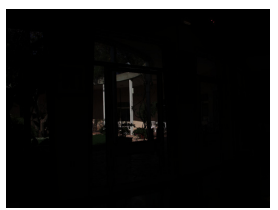

(a)

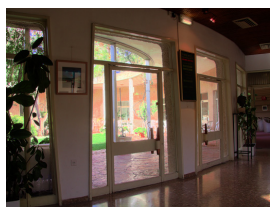

(e)

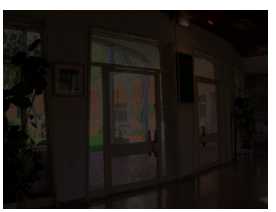

(b)

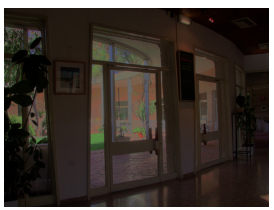

(f)

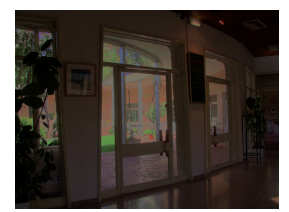

(c)

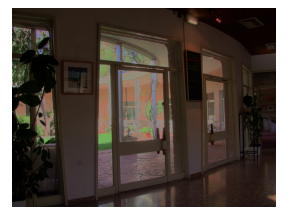

(g)

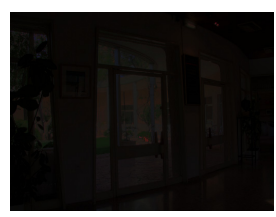

(d)

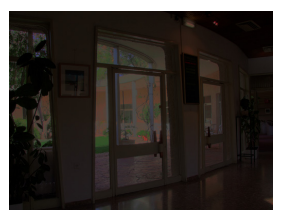

(h)

Fig. 3. Drago's TMO application to different luminance channels: (a) linearly mapped original image, (b) Y of YUV, (c) PCA, (d) G of RGB, (e) V of HSV, (f) I of HSI, (g) L of HSL, (h) L of Lab

TMO's best results for different luminance channels and Fig. 4 shows the KW test box plots. The best improvements for Mantiuk's TMO and Drago's TMO were achieved by applying them to HSV's V channel. This can be explained by the fact that V's value is always higher or equal to values of other channels.

\subsection{The Proposed Channel}

Drago's TMO and Mantiuk's TMO showed a much greater flexibility then Durand's TMO. Therefore the best TMQI calculation on selected images was repeated for Drago's and Mantiuk's TMO extended by the proposed luminance channel calculation norm $p$, which was treated as an additional parameter with values from $\{1,2, \ldots, 100\}$. The best TMQIs for Durand's TMO, Mantiuk's TMO, Drago's TMO, and additionally Reinhard's global TMO [15] all applied to the YUV's Y channel were compared to best TMQIs for Mantiuk's TMO and Drago's TMO applied to the proposed channel with variable parameter $p$ for every image. Reinhard's TMO intensity parameter value was from $\{-8.0,-7.9, \ldots, 8.0\}$ and the values of the both adapt parameters were from $\{0.0,0.1, \ldots, 1.0\}$.

Table 2 shows the statistics of the best TMQIs. The application of Drago's TMO to the proposed channel resulted in a $17 \%$ increase of the mean of the best TMQI (25\% for 20 images with the highest dynamic range and $9 \%$ for 20 images with the lowest dynamic range), while the application of Mantiuk's TMO to the proposed channel resulted in in a 10\% increase of the mean TMQI (18\% for images with the highest dynamic range and $3 \%$ for images with the lowest dynamic range). Drago $p$ is shown to be as good as Reinhards's TMO at the cost of extension by a new parameter. Fig. [5 gives the best results of different TMOs. 


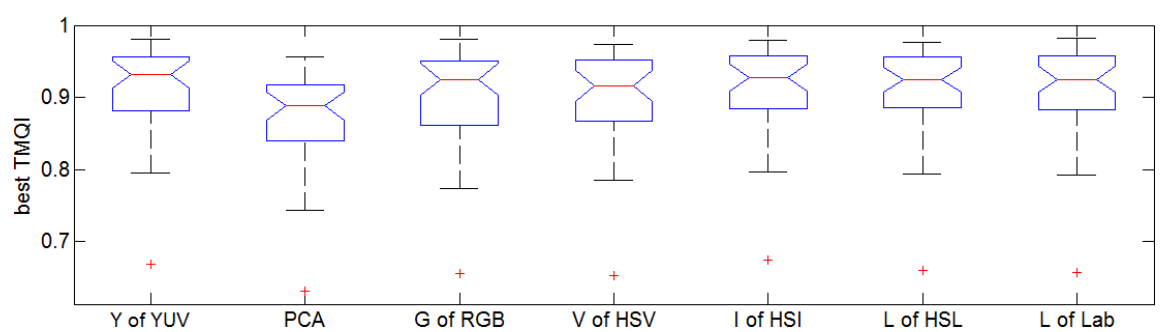

(a)

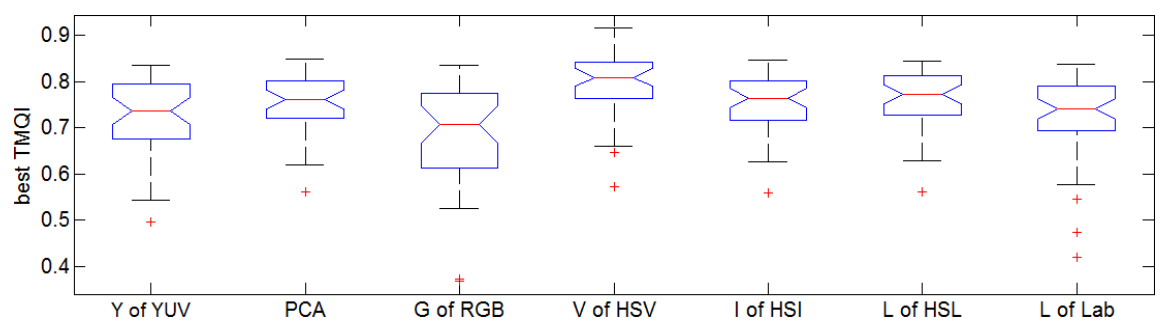

(b)

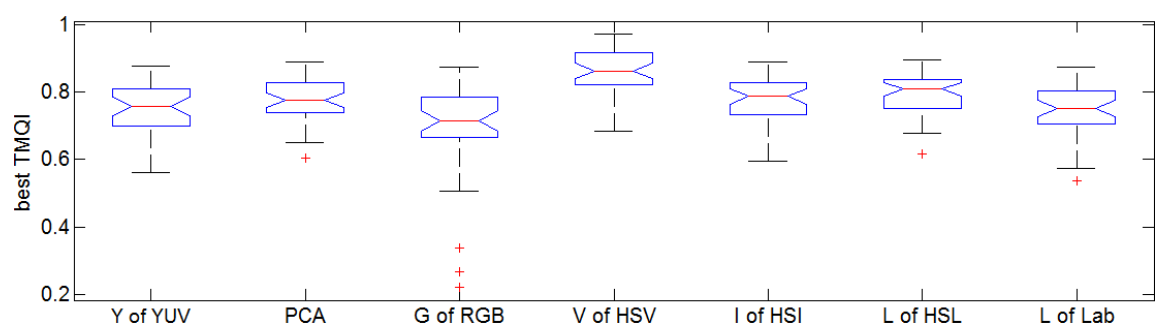

(c)

Fig. 4. Kruskal-Wallis different luminance channels box plots for different TMOs: (a) Durand's TMO, (b) Mantiuk's TMO, (c) Drago's TMO

Table 2. The best TMQI for several TMOs on selected images (higher is better)

\begin{tabular}{|c|c|c|c|c|c|c|}
\hline & Durand & Reinhard & Mantiuk & Mantiuk $_{p}$ & Drago & Drago $_{p}$ \\
\hline mean & 0.9125 & 0.8789 & 0.7286 & 0.8068 & 0.7521 & 0.8803 \\
\hline median & 0.9325 & 0.8874 & 0.7367 & 0.8134 & 0.7571 & 0.8915 \\
\hline
\end{tabular}

The KW test for the best TMQIs achieved when Drago's TMO was applied to HSV's V channel and for the best TMQIs achieved for Drago ${ }_{p}$ detected no statistically significant difference. The KW test was also performed for the best results of Durand's TMO and Drago's TMO in the first case and for the best results of Durand's TMO and Drago $_{p}$ in the second case. Unlike the first test, the second test resulted in the KW $p$ value above 0.01 meaning a less significant difference. For Mantiuk's TMO $p$ was below 0.01 for both cases. 


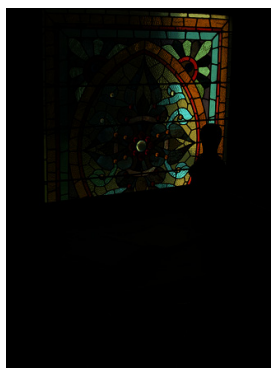

(a)

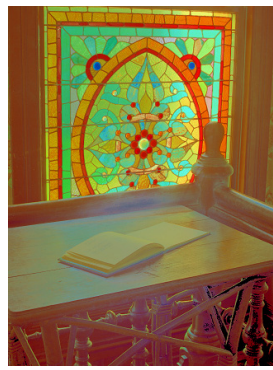

(e)

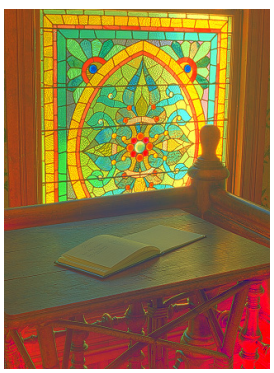

(b)

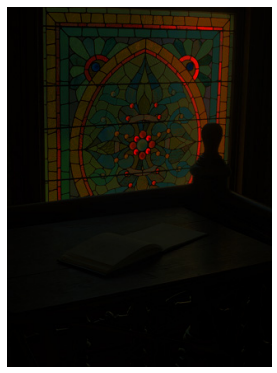

(f)

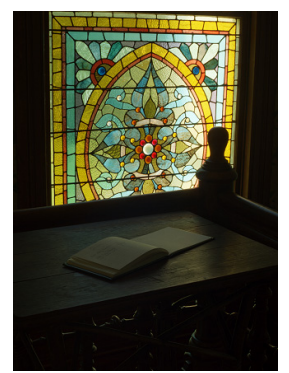

(c)

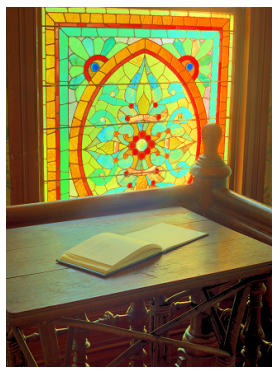

(g)

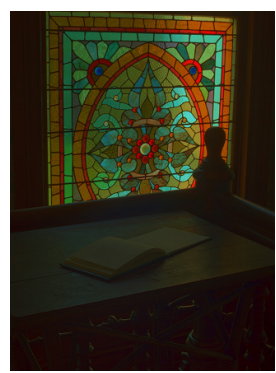

(d)

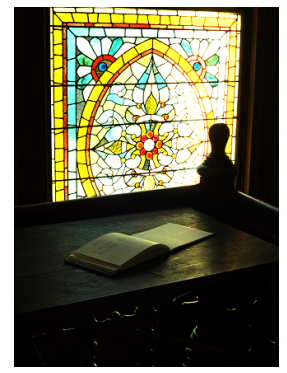

(h)

Fig. 5. Different tone mapping for the same HDR image: (a) linear mapping, (b) Du-

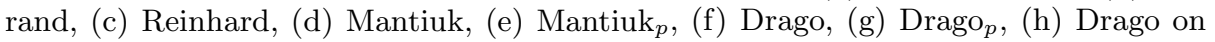
$\mathrm{V}$ of HSV

\section{Conclusions and Future Work}

The proposed definition of the luminance channel has a significant impact on the TMO's resulting image quality and it achieved the best statistically significant results in terms of quality by adding an additional parameter. From the wellknown luminance channels the best results were achieved with the HSV's V channel. TMOs previously achieving lower quality were transformed into TMOs whose quality compares well with TMOs that produce high quality images. In future other suitable and adjustable luminance channels should be researched.

Acknowledgments. The authors would like to thank Dr. Tomislav Petković for his useful remarks. This research has been partially supported by the European Union from the European Regional Development Fund by the project IPA2007/HR/16IPO/001-040514 "VISTA - Computer Vision Innovations for Safe Traffic." 


\section{References}

1. HDR imaging (2014), http://docs.opencv.org/master/modules/photo/doc/hDR_imaging.html

2. Braun, G.J., Fairchild, M.D.: Image lightness rescaling using sigmoidal contrast enhancement functions. Journal of Electronic Imaging 8(4), 380-393 (1999)

3. Drago, F., Myszkowski, K., Annen, T., Chiba, N.: Adaptive logarithmic mapping for displaying high contrast scenes. In: Computer Graphics Forum, vol. 22, pp. 419-426. Wiley Online Library (2003)

4. Durand, F., Dorsey, J.: Fast bilateral filtering for the display of high-dynamic-range images. ACM Transactions on Graphics (TOG) 21(3), 257-266 (2002)

5. Fattal, R., Lischinski, D., Werman, M.: Gradient domain high dynamic range compression. ACM Transactions on Graphics (TOG) 21, 249-256 (2002)

6. Jarque, C.M., Bera, A.K.: A test for normality of observations and regression residuals. International Statistical Review/Revue Internationale de Statistique, 163-172 (1987)

7. Koschan, A., Abidi, M.: Digital Color Image Processing. Wiley (2008), http://books.google.hr/books?id=SlXgTyQ86VsC

8. Kuang, J., Yamaguchi, H., Johnson, G.M., Fairchild, M.D.: Testing HDR image rendering algorithms. In: Color and Imaging Conference, vol. 2004, pp. 315-320. Society for Imaging Science and Technology (2004)

9. Kuang, J., Yamaguchi, H., Liu, C., Johnson, G.M., Fairchild, M.D.: Evaluating HDR rendering algorithms. ACM Transactions on Applied Perception (TAP) 4(2), 9 (2007)

10. Larson, G.W., Rushmeier, H., Piatko, C.: A visibility matching tone reproduction operator for high dynamic range scenes. IEEE Transactions on Visualization and Computer Graphics 3(4), 291-306 (1997)

11. Mantiuk, R., Daly, S., Kerofsky, L.: Display adaptive tone mapping. ACM Transactions on Graphics (TOG) 27, 68 (2008)

12. Maxwell, S., Delaney, H.: Designing Experiments and Analyzing Data: A Model Comparison Perspective. No. s. 1 in Designing Experiments and Analyzing Data: A Model Comparison Perspective, Lawrence Erlbaum Associates (2004), http://books.google.hr/books?id=gKZbD3lL88AC

13. Meylan, L., Susstrunk, S.: High dynamic range image rendering with a retinexbased adaptive filter. IEEE Transactions on Image Processing 15(9), 2820-2830 (2006)

14. NTUST Compute Graphics Group: HDR (2014), http://graphics.csie.ntust.edu.tw/pub/HDR/

15. Reinhard, E., Devlin, K.: Dynamic range reduction inspired by photoreceptor physiology. IEEE Transactions on Visualization and Computer Graphics 11(1), 13-24 (2005)

16. Reinhard, E., Heidrich, W., Debevec, P., Pattanaik, S., Ward, G., Myszkowski, K.: High dynamic range imaging: Acquisition, display, and image-based lighting. Morgan Kaufmann (2010)

17. Reinhard, E., Stark, M., Shirley, P., Ferwerda, J.: Photographic tone reproduction for digital images. ACM Transactions on Graphics (TOG) 21, 267-276 (2002)

18. Yeganeh, H., Zhou, W.: Objective Quality Assessment of Tone Mapped Images. IEEE Transactions on Image Processing 22(2), 657-667 (2013) 